\title{
RELATION OF CONE BEAM COMPUTED TOMOGRAPHY ASSESSMENT OF MANDIBULAR BONE DENSITY TO DUAL ENERGY X-RAY ABSORPTIOMETRY IN TYPE 2 DIABETES MELLITUS PATIENTS
}

\author{
Lobna M. El Saadawy ${ }^{1} B D S$, Rania A. Fahmy² $P h D$, Khaled A. Matrawy ${ }^{3} P h D$, Mohammed \\ H. Zeitoun ${ }^{4} P h D$, Yousria S. Gaweesh ${ }^{5} P h D$.
}

\begin{abstract}
INTRODUCTION: One of the complications associated with diabetes mellitus is decrease in bone mineral density (BMD). Therefore, quantification of BMD for these patients before a dental implant procedure is essential. Cone beam computed tomography (CBCT) is an important tool that can be used for qualitative and quantitative bone assessment for treatment planning of dental implants.

OBJECTIVES: Evaluation of mandibular bone mineral density measured by CBCT and its correlation to T-score obtained by dual energy xray absorptiometry (DEXA) in relation to blood glycemic control as detected by glycosylated hemoglobin (HbA1c) in type 2 diabetic patients (T2DM).

MATERIALS AND METHODS: The study was conducted clinically on twenty-four patients having T2DM, referred for CBCT examination as a part of implant planning. BMD was measured for each edentulous mandibular space using CBCT scan. The patients were grouped according to the level of HbA1c into 4 groups; control group, well-controlled group, moderately controlled group and poorly controlled group. Additionally, each candidate was examined radio-graphically using DEXA. Then, a statistical correlation between HbA1c values in relation to mandibular BMD values and T-score values was investigated.

RESULTS: there was a statistically significant correlation between HbA1c values and BMD variables including: mandibular edentulous quad BMD and T-score values ( $\mathrm{p}<0.001$ ), and a statistically significant correlation between T-score value and Mandibular edentulous quad BMD $(\mathrm{p}<0.001)$.

CONCLUSIONS: Osteoporosis is a commonly underestimated problem in diabetes patients. Osteoporosis can be predicted using CBCT, so it's the dentist responsibility to refer osteoporotic patients once being suspected.

KEYWORDS: CBCT, BMD, DEXA, type 2 diabetes, Osteoporosis.

1. Instructor in Oral Medicine, Oral Periodontology, Oral Diagnosis and Oral Radiology, Faculty of Dentistry, Alexandria University.

2. Lecturer of Oral Medicine, Oral Periodontology, Oral Diagnosis and Oral Radiology.

3. Assistant Professor of Diagnostic Radiology, Medical Research Institute.

4. Lecturer of Internal Medicine, Faculty of Medicine.

5. Professor of Oral Medicine, Oral Periodontology, Oral Diagnosis and Oral Radiology.
\end{abstract}

Corresponding author:

E-mail: dr.lobnaelsaadawy@gmail.com

\section{INTRODUCTION}

Diabetes is an enormous and growing clinical and public health obstacle. In 2015, the International Diabetes Federation (IDF) estimated that 415 million adults had diabetes worldwide, and that, by 2040, the number will increase to 642 million (1). While as for Middle East and North Africa; the country with the largest number of adults with diabetes is Egypt with an average of 7.8 million people as estimated by the IDF in 2015 (2).

Previously, the list of target organs affected by type 2 diabetes mellitus (T2DM) did not include the skeletal system. However recently, diabetes mellitus has been shown to be associated with some complications affecting the skeleton; including decrease in bone mineral density that may be associated with osteopenia or osteoporosis, and impaired bone regeneration potential $(3,4)$.

The quantification of body bone mineral density (BMD) for these patients is therefore essential. Dual-energy x-ray absorptiometry (DEXA) is considered the "gold standard" for noninvasive measurement of bone mineral density in orthopedics, endocrinology and traumatology (5).

Decreased bone mineral density and osteoporosis can be considered as a risk factor for reduced mandibular alveolar bone density i.e. mandible might be affected with osteoporosis (6). Mandibular bone quality and quantity can be detected using different oral and maxillofacial techniques which were found indispensable in dental implants treatment planning (7).

Recently, cone beam computed tomography (CBCT) has been increasingly replacing other oral and maxillofacial techniques. It is a very promising technique relevant to dental implants planning, providing 3-dimensional evaluation and offering lower cost as compared to CT technology $(8,9)$.

The aim of this study was to evaluate mandibular bone density in patients with T2DM as detected by CBCT technique. Additionally, to correlate the obtained mandibular bone density values with the diabetic condition 
as assessed by HbA1c and T-score values as obtained by DEXA scan.

\section{MATERIALS AND METHODS}

The study was performed in the Faculty of Dentistry, Alexandria University after gaining the approval of the Research Ethics Committee. Twenty four patients randomly allocated from the outpatient clinic at the department of Oral Medicine, Periodontology, Oral Diagnosis, and Oral Radiology, Faculty of Dentistry and the department of Internal Medicine, Faculty of Medicine, Alexandria University, who will require a CBCT examination as a part of their dental implant planning. All patients signed informed consents for the agreement to participate in the study.

The patients who presented with smoking and/ or alcoholism, patients suffering from any systemic disease affecting bone mineral density other than T2DM for the diabetic groups were excluded from the study. Additionally, patients on any medication affecting bone mineral density other than oral anti-diabetic drugs for the diabetic groups, or, those presenting with any bone lesions, fractures, deformity, or any previous surgeries within the edentulous area, together with lactating, pregnant or menopausal female patients were excluded from the study.

The entire study sample was subjected to blood sampling by a venipuncture of the antecubital vein in order to determine glycated hemoglobin (HbA1c) values. According to the HbA1c level, the patients were grouped into four groups, each group included 6 patients; Group A: Normal; non-diabetic individuals (control group) have (HbA1c $\leq 6.0 \%)$. Group B: individuals with type 2 diabetes stratified as well-controlled (HbA1c 6.1-8.0\%), Group C: moderately controlled (HbA1c 8.1-10.0\%), Group D: poorly controlled (HbA1c $\geq 10.1 \%)(7,10-12)$.

DEXA technique was used to evaluate the whole body bone mineral density that was evaluated using T-score with standard curves (13).

CBCT technique was used to assess the mandibular bone density related to the edentulous bounded saddle. Before performing any measurements, a precise panoramic curve was accurately drawn on a selected axial cut at approximately the mid-tooth level of the mandibular teeth (14). The inferior alveolar nerve canal was accurately traced until the mental foramen using the panoramic image.

Using the reformatted panoramic image, obtained from the CBCT scan, a specific cross- sectional cut was obtained to achieve bone density measurements, as follows:

1. In case of a single missing posterior tooth within the bounded saddle, the mesio-distal dimension of the bounded saddle was calculated and halved. A cross-sectional cut going by this exact mid- distance was selected to perform the bone density measurements (Fig.1).

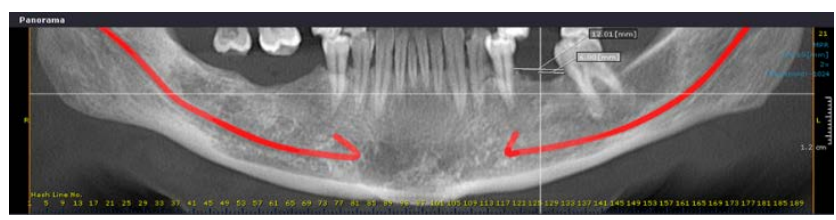

Figure 1: In case of a single missing posterior tooth, as in the mandibular left quad of this case, the mesio-distal dimension of the bounded saddle was calculated and halved.
2. In case of multiple missing posterior teeth, the total mesiodistal dimension of the bounded edentulous area was measured. Then the mesio-distal dimension for the region representing each missing tooth was calculated by dividing the total mesio-distal dimension by the number of missing teeth on the reformatted panoramic image. Next, the approximate width of each missing tooth region was halved. A cross-sectional cut going by the exact mid- distance of each missing tooth region's width was selected to perform the bone density measurements (Fig.2).

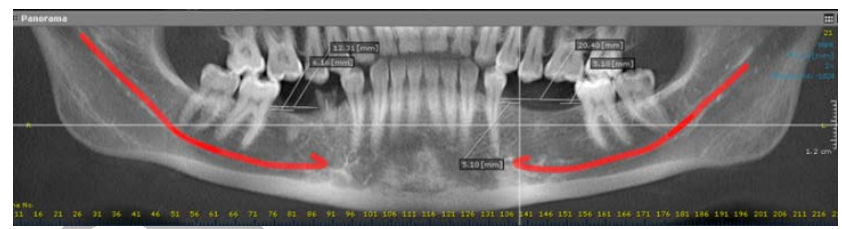

Figure 2: In case of multiple missing posterior teeth, as in the mandibular left quad of this case, the mesio-distal dimension for the region representing each missing tooth was calculated and halved.

Using the obtained cross-sectional cut at the middle of each edentulous area representing a missing tooth, trabecular bone density was obtained using the region of interest measuring tool (ROI) (15) (Fig.3). Cross-sectional slice thickness and measured area size was standardized in all cases. Cone beam computed tomography radiological density of each posterior bounded saddle was considered as the mean of all calculated gray values for each missing tooth region within the bonded saddle (16).

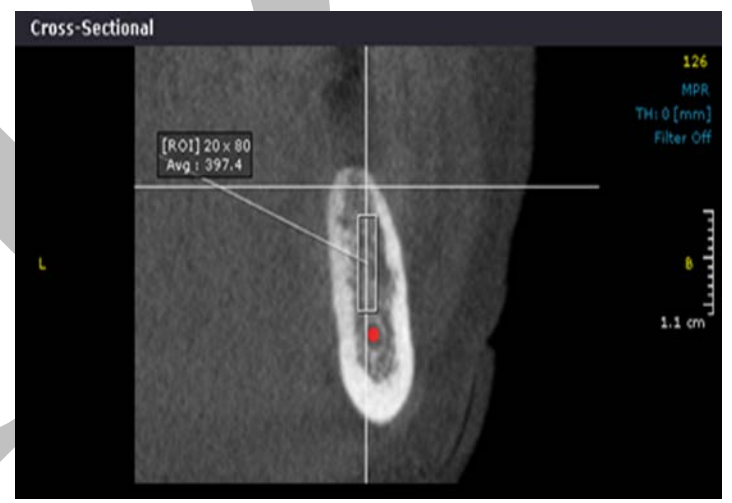

Figure 3: On the selected cross-sectional image, the average mandibular trabecular bone density of the edentulous space was calculated.

\section{Statistical analysis}

The difference in the mean T-score values and the mean mandibular edentulous quad BMD were estimated using Chi square test for comparing between the four groups. Significance between groups was done using Post Hoc Test (Dunn's multiple comparisons test).

Correlation between HbA1c values with T- score and mandibular edentulous quad BMD was obtained using Pearson coefficient. Additionally, correlation between Tscore and mandibular edentulous quad BMD was obtained using Spearman coefficient.

A [P] value less than 0.05 was considered significant. The values were given as a mean value \pm SD (standard deviation). 


\section{RESULTS}

Twenty four patients were included in this study with an age range from 40 to 55 years. Fourteen patients (58.3\%) were males and ten patients (41.6\%) were females.

\section{Radiographic Results}

Comparisons between the four studied groups according to $\mathrm{T}$ - score and mandibular edentulous quad BMD are shown in Table 1. On comparing T-score values, group D showed a statistically significant difference $(p<0.05)$ in comparison to other study groups.

Table 1: Comparison between the four studied groups according to $\mathrm{T}$ - score and mandibular edentulous quad BMD.

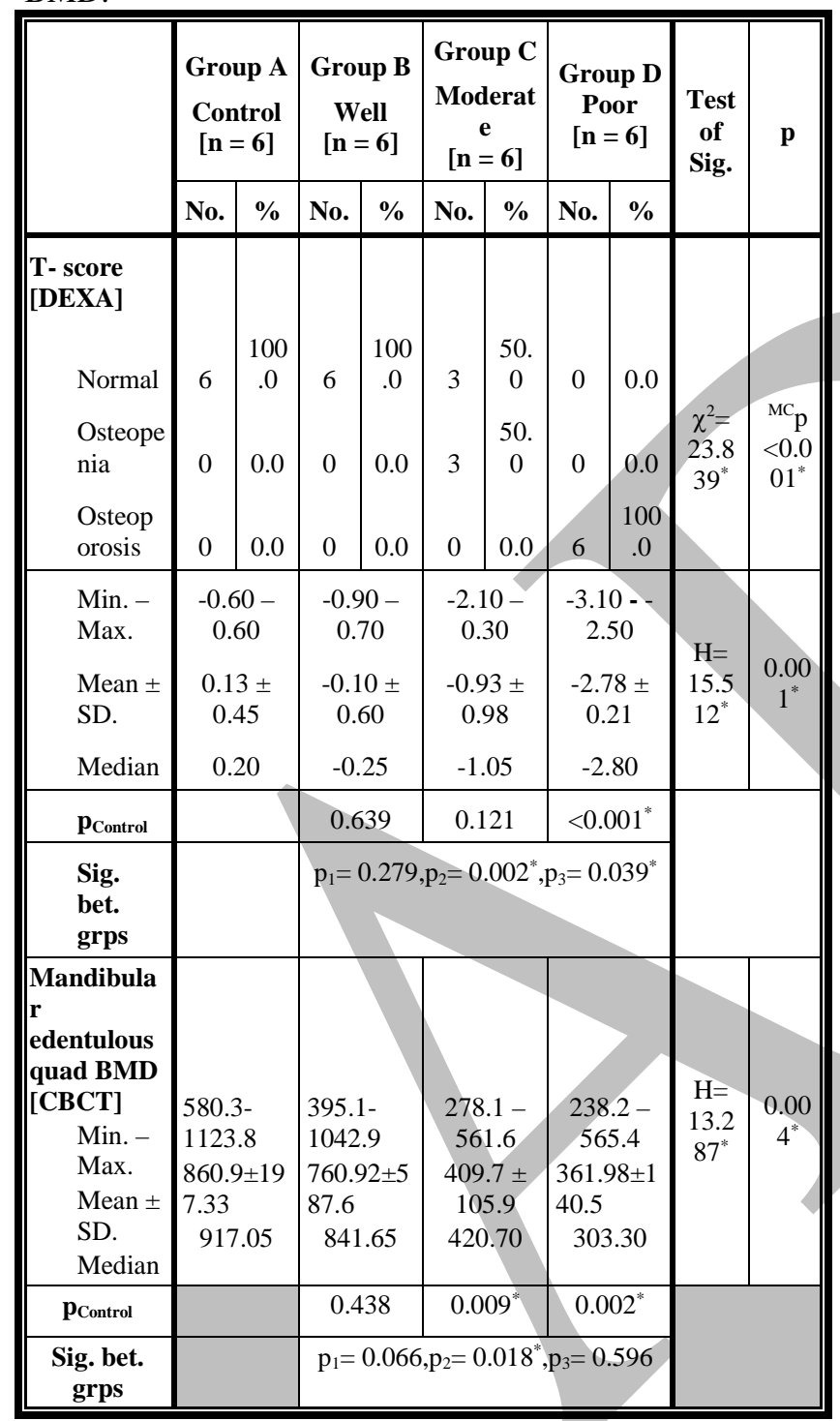

$\chi^{2}$, p: $\chi^{2}$ and $\mathrm{p}$ values for Chi square test for comparing between the four groups

${ }^{\mathrm{MC}} \mathrm{p}$ : $\mathrm{p}$ value for Monte Carlo for Chi square test for comparing between the four groups

$\mathrm{H}$, p: $\mathrm{H}$ and p values for Kruskal Wallis test, Sig. bet. grps was done using Post Hoc Test [Dunn's multiple comparisons test]

pcontrol: p value for comparing between control with each other groups

$\mathrm{p}_{1}$ : $\mathrm{p}$ value for comparing between well and moderately

p2: $\mathrm{p}$ value for comparing between well and poorly

$\mathrm{p}_{3}$ : $\mathrm{p}$ value for comparing between moderately and poorly

*: Statistically significant at $\mathrm{p} \leq 0.05$
However, on comparing mandibular edentulous quad BMD values, group A and group B showed comparable mean values. While, group $C$ as well as group $D$ showed significantly higher values than control group.

\section{Correlation results}

The present results showed that, there is a statistically significant negative correlation between HbA1c values and BMD variables including; T-score values $(\mathrm{p}<0.001)$ and mandibular edentulous quad BMD ( $<<0.001)$ (Table 2).

Study results showed a statistically significant correlation between $\mathrm{T}$ - score values and mandibular edentulous quad BMD values $(\mathrm{p}<0.001)$ (Table 3$)$.

Table 2: Correlation between HbA1c with density parameters in the total study sample.

\begin{tabular}{|l|c|c||}
\hline \multirow{2}{*}{} & \multicolumn{2}{|c|}{ HbA1c } \\
\cline { 2 - 3 } & $\mathbf{r}$ & $\mathbf{p}$ \\
\hline T- score & $-0.830^{*}$ & $<0.001^{*}$ \\
\hline Mandibular edentulous quad BMD & $-0.758^{*}$ & $<0.001^{*}$ \\
\hline
\end{tabular}

r: Pearson coefficient

*: Statistically significant at $\mathrm{p} \leq 0.05$

Table 3: Correlation between T- score and Mandibular edentulous quad BMD in the total study sample.

\begin{tabular}{|l|c|c|}
\hline \multirow{2}{*}{} & \multicolumn{2}{|c|}{ T- score } \\
\cline { 2 - 3 } & $\mathbf{r}_{\mathbf{s}}$ & $\mathbf{p}$ \\
\hline $\begin{array}{l}\text { Mandibular edentulous quad } \\
\text { BMD }\end{array}$ & $0.790^{*}$ & $<0.001^{*}$ \\
\hline
\end{tabular}

rs: Spearman coefficient

*: Statistically significant at $\mathrm{p} \leq 0.05$

\section{DISCUSSION}

Preoperative assessment of both bone quality and quantity is essential to assist the clinician with planning for implant therapy. Accurate qualitative and quantitative information on the bone will help the surgeon identify suitable implant site, thereby improving the surgical planning and eventually, the success rate of the procedure. To obtain this knowledge preoperative, adequate radiographic examination is required. CBCT imaging and the interactive software enable bone quality and quantity measurements on CBCT slices $(17,18)$. This study aimed at correlating Tscore values obtained by DEXA and mandibular BMD values obtained by CBCT for T2DM patients in relation to their HbA1c values.

The current study presented a statistically nonsignificant difference between non-diabetic subjects (control) versus good and moderate control diabetic subjects $(\mathrm{p}>0.05)$ in relation to T- score, and a statistically significant difference between non-diabetic (control) subjects versus poor control diabetic subjects $(p \leq 0.05)$ in relation to $\mathrm{T}$-score results. In other words, poor control diabetic subjects showed lower BMD as shown by T-score values in relation to other study groups.

Furthermore, study results demonstrated a negative correlation between HbA1c values and T-score values as measured by DEXA. In explanation, high levels of HbA1c correspond to low levels of skeletal BMD.

Agreeing results were shown by Adel et al. (19), who compared a type 2 diabetic group to a healthy control group. 
Their results showed that BMD values were found to be significantly low in terms of the lumbar T-scores in type 2 diabetes group. The study results, also, went along with the results of Karimifar et al. (20), who reported that osteopenia and osteoporosis is more common in diabetic postmenopausal women compared to nondiabetic postmenopausal women.

Contradictory studies compared non-diabetic subjects to diabetic subjects in relation to femoral neck and lumbar spine BMD measured by DEXA, concluded that subjects with T2DM have a higher BMD (21-23).

These conflicting study results could be explained by the fact that HbA1c levels measured at the time when BMD measurements were performed reflected only short term glycemic control (not beyond 3 months); specifically, it is difficult to make sure about the duration of glycemic control from a single test. Therefore, investigation of serial HbA1c levels for longer periods of time for BMD correlation would be more accurate.

On the other hand, Majima et al. (24) reported lower BMD values in type 2 diabetic population in comparison to a non-diabetic population, after a follow up for HbA1c values for a period of two years. These results go along with the current study results; although, serial $\mathrm{HbA1c}$ measurements were taken throughout 2 years before BMD measurements.

Higher BMD associated with poor metabolic control in type 2 diabetic patients could, also, be explained by their hyperinsulinemia. This is because insulin has an anabolic effect on bone (25), where, insulin binds to receptors on osteoblasts and stimulates bone formation (26), suggesting that insulin may be a physiological antagonist of bone resorption. Type 1 diabetes mellitus and late stages of type 2 diabetes mellitus show reduced insulin levels and reduced insulin signaling in osteoblasts, which may negatively affect bone and contribute to reduced bone formation caused by diabetes (27).

Poor glycemic control was not associated with high insulin levels in the Majima et al. study, it didn't therefore lead to higher BMD (24). Accordingly, different insulin levels in type 2 diabetic patients, high in new-onset diabetes and low in long-standing cases, might be one of the explanations for the contradictory study results (28). In the current study, all new-onset diabetes cases were excluded by a strict inclusion criterion of accepting cases diagnosed with T2DM since at least 5 years.

Furthermore, the present study presented a statistically non-significant difference between non-diabetic subjects (control) versus good control diabetic subjects $(p>0.05)$ in relation to mandibular edentulous quad BMD. In conclusion, good control T2DM had no effect on mandibular BMD.

Similar results for a study addressing the mandibular BMD were obtained by Jolly et al. (29), who compared the jaws' bone density in non-diabetic and good control type II diabetes patients (HbA1c level between 6.1-8\%) using spiral computed tomography. Bone densities at trabecular, buccal, and lingual cortical regions of maxillary and mandibular edentulous arches were measured. This study showed no significant changes in bone density between the controlled diabetic and non-diabetic subjects, concluding that jaws' bone density does not seem to be affected in controlled type II diabetics.
Similarly, Javed et al. (30) reported successful dental implant osseo-integration in subjects with good control diabetes in a similar manner as in non-diabetic subjects. These results were clarified by that metabolic control is essential for osseointegration to take place, as constant hyperglycaemia delays healing of the bone around the implants (31).

These results were explained by evidence that glycemic control is crucial for alleviating the risk of bone fragility and fracture in T2DM. Adequate glycemic control reduces the risk of micro and macro-vascular complications. Diabetic vascular complications may directly contribute to bone fragility as well as increase an individual's risk of falls (32).

The current study presented a statistically significant difference between non-diabetic subjects (control) and moderate and poor control diabetic subjects $(\mathrm{p} \leq 0.05)$ in relation to mandibular edentulous quad BMD.

Similar results were achieved by Onoyama et al. (33), who conducted a study addressing the effect of T2DM on mandibular bone in GK diabetic rats in comparison to nondiabetic Wistar rats (control group). GK diabetic rats showed a reduced medullary BMD in the mandibular molar area, regional morphological changes, and a reduction in bone hardness.

Furthermore, Nemtoi et al. (7) compared a diabetic to a non-diabetic patient group in relation to mandibular BMD as measured by CBCT. Their study demonstrated a significant inverse correlation between the values of trabecular bone density in the posterior region of the mandible and $\mathrm{HbA1c}$; with poor control diabetes showing lowest BMD values in the posterior region of the mandible.

Contradicting results were achieved by another study discussing the relationship between T2DM (mean HbA1c values was $8.8 \pm 2.5$ ) and mandibular changes, using panoramic radiography; these results illustrated no difference between T2DM patients and healthy subjects with regard to mandibular BMD values (34). The findings might have resulted due to measuring BMD values from the angle of the mandible, which might not reflect the values of the dento-alveolar region. However, they referred the reason for these contradictory results to the effect of hyperinsulinaemia.

This study signified the importance of the CBCT in the detection of changes in BMD and demonstrated that CBCT is an effective tool in the diagnosis of osteoporosis. Where, a positive correlation was detected between T-score values as measured by DEXA and grey values measured by CBCT in edentulous mandibular quad.

These results are in accordance with Barngkgei et al. (13) who, compared menopausal and postmenopausal women receiving DEXA and CBCT scans. They concluded that osteoporosis can be predicted with a high accuracy from the radiographic density value of the mandibular body. Similarly, Güngör et al. (35) observed a positive correlation between measurements of spine BMD as assessed by DEXA and right and left mandibular density values as assessed by CBCT.

Furthermore, Guerra et al. (36) systematically reviewed the literature about the capability of CBCT images to identify individuals with low BMD. They concluded that CBCT-derived radiographic density is a promising tool for differentiating individuals with osteoporosis from individuals with normal BMD. 
On the other hand, in an in-vitro study, Hua et al. (37) compared DEXA derived BMD values and the CBCTderived density for the mandible and found no correlation. However, these selected studies have used different CBCT devices and Voxel sizes. Some authors have verified that the trabecular bone measurements, and consequently the image quality, are affected by technical parameters, such as the Voxel size, the unit itself, tube voltage and amperage, and FOV selection (38).

\section{CONCLUSION}

These results indicate decrease in BMD in type 2 diabetes and suggest the importance of keeping good metabolic control to prevent osteoporosis in type 2 diabetic patients. CBCT-derived radiographic density using the CBCTviewer software can be promising for screening BMD in future studies. In cases where the CBCT is indicated, dentists could have an important role in identifying patients with low BMD.

\section{CONFLICT OF INTEREST}

The authors declare that they have no conflicts of interest.

\section{REFERENCES}

1. Herman WH. The Global Burden of Diabetes: An Overview. In: Jack SD (ed). Diabetes Mellitus in Developing Countries and Underserved Communities. USA: Springer; 2017. p 1-5.

2. International Diabetes Federation. IDF diabetes atlas. 7th ed. Brussels International Diabetes Federation; 2015.

3. Vestergaard P. Discrepancies in bone mineral density and fracture risk in patients with type 1 and type 2 diabetes--a meta-analysis. Osteoporos Int. 2007;18:427-44.

4. Schwartz AV, Garnero P, Hillier TA, Sellmeyer DE, Strotmeyer ES, Feingold KR, et al. Pentosidine and increased fracture risk in older adults with type 2 diabetes. J Clin Endocrinol Metab. 2009;94:2380-6.

5. Marquezan M, Lau TCL, Mattos CT, Cunha ACd, Nojima LI, Sant'Anna EF, et al. Bone mineral density. Angle Orthod. 2012;82:62-6.

6. Amam A, Rustom J. Assessment of Mandibular Alveolar Bone Density in Osteoporotic Adults in Syria. J Dent Oral Med. 2014;2:26-32.

7. Nemtoi A, Ladunca O, Dragan E, Budacu C, Mihai C, Haba D. Quantitative and qualitative bone assessment of the posterior mandible in patients with diabetes mellitus: a cone beam computed tomography study. Rev Med Chir Soc Med Nat Iasi. 2013;117:1002-8.

8. Suomalainen A, Pakbaznejad Esmaeili E, Robinson S. Dentomaxillofacial imaging with panoramic views and cone beam CT. Insights Imaging. 2015;6:1-16.

9. Monsour PA, Dudhia R. Implant radiography and radiology. Aust Dent J. 2008;53 (Suppl 1):S11-25.

10. Oates TW, Dowell S, Robinson M, McMahan CA. Glycemic control and implant stabilization in type 2 diabetes mellitus. J Dent Res 2009;88:367-71.

11. Gomez-Moreno G, Aguilar-Salvatierra A, Rubio Roldan J, Guardia J, Gargallo J, Calvo-Guirado JL. Peri-implant evaluation in type 2 diabetes mellitus patients: a 3-year study. Clin Oral Implants Res. 2015;26:1031-5.
12. Dowell S, Oates TW, Robinson M. Implant success in people with type 2 diabetes mellitus with varying glycemic control: a pilot study. J Am Dent Assoc 2007;138:355-61.

13. Barngkgei I, Al Haffar I, Khattab R. Osteoporosis prediction from the mandible using cone-beam computed tomography. Imaging Sci Dent. 2014;44:263-71.

14. Kenawy S, ElBeshlawy D, Dahaba M. Quantitative and qualitative maxillary jaw bone assessment using cone beam computed tomography of a diabetic versus a nondiabetic sample of egyptian population. IJAR. 2017;5:2113-26.

15. Turkyilmaz I, McGlumphy EA. Influence of bone density on implant stability parameters and implant success: a retrospective clinical study. BMC Oral Health. 2008;8:32.

16. Parsa A, Ibrahim N, Hassan B, Stelt P, Wismeijer D. Bone quality evaluation at dental implant site using multislice CT, micro-CT, and cone beam CT. Clin Oral Implants Res 2015;26:e1-7.

17. Gerlach NL, Meijer GJ, Borstlap WA, Bronkhorst EM, Bergé SJ, Maal TJJ. Accuracy of bone surface size and cortical layer thickness measurements using cone beam computerized tomography. Clin Oral Implants Res 2013;24:793-7.

18. Aranyarachkul P, Caruso J, Gantes B, Schulz E, Riggs M, Dus I, et al. Bone density assessments of dental implant sites: 2. Quantitative cone-beam computerized tomography. Int J Oral Maxillofac Implants. 2005;20:41624.

19. Adil C, Aydin T, Taspinar O, Kiziltan H, Eris AH, Hocaoglu IT, et al. Bone mineral density evaluation of patients with type 2 diabetes mellitus. J Phys Ther Sci. 2015;27:179-82.

20.Karimifar M, Pasha MAP, Salari A, Zamani A, Salesi M, Motaghi P. Evaluation of bone loss in diabetic postmenopausal women. J Res Med Sci. 2012;17:1033-8.

21. de $L$, II, van der Klift $M$, de Laet CE, van Daele PL, Hofman A, Pols HA. Bone mineral density and fracture risk in type-2 diabetes mellitus: the Rotterdam Study. Osteoporos Int. 2005;16:1713-20.

22. Leidig-Bruckner G, Grobholz S, Bruckner T, ScheidtNave C, Nawroth P, Schneider JG. Prevalence and determinants of osteoporosis in patients with type 1 and type 2 diabetes mellitus. BMC Endocr Disord. 2014;14:33.

23. Bayani MA, Karkhah A, Hoseini SR, Qarouei R, Nourodini HQ, Bijani A, et al. The Relationship Between Type 2 Diabetes Mellitus and Osteoporosis in Elderly People: a Cross-sectional Study. IBBJ. 2016;2:39-46.

24. Majima T, Komatsu Y, Yamada T, Koike Y, Shigemoto M, Takagi C, et al. Decreased bone mineral density at the distal radius, but not at the lumbar spine or the femoral neck, in Japanese type 2 diabetic patients. Osteoporos Int. 2005;16:907-13.

25. Ivaska KK, Heliövaara MK, Ebeling P, Bucci M, Huovinen V, Väänänen $\mathrm{HK}$, et al. The effects of acute hyperinsulinemia on bone metabolism. Endocr Connect. 2015;4:155-62.

26. Thomas D, Hards D, Rogers S, Ng K, Best J. Insulin receptor expression in bone. $\mathrm{J}$ Bone Miner Res. 1996;11:1312-20.

27. Jiao H, Xiao E, Graves DT. Diabetes and its effect on bone and fracture healing. Curr Osteoporos Rep. 2015;13:32735.

28. Stolk R, Van Daele P, Pols H, Burger H, Hofman A, Birkenhäger J, et al. Hyperinsulinemia and bone mineral 
density in an elderly population: The Rotterdam Study. Bone. 1996;18:545-9.

29. Jolly SJ, Hegde C, Shetty NS. Assessment of Maxillary and Mandibular Bone Density in Controlled Type II Diabetes: A Computed Tomography Study. J Oral Implantol. 2015;41:400-5

30. Javed F, Romanos GE. Impact of diabetes mellitus and glycemic control on the osseointegration of dental implants: a systematic literature review. J Periodontol. 2009;80:1719-30.

31. Mellado-Valero A, Ferrer Garcia JC, Herrera Ballester A, Labaig Rueda C. Effects of diabetes on the osseointegration of dental implants. Med Oral Patol Oral Cir Bucal. 2007;12:E38-43.

32. Adil M, Khan RA, Kalam A, Venkata SK, Kandhare AD, Ghosh P, et al. Effect of anti-diabetic drugs on bone metabolism: evidence from preclinical and clinical studies. Pharmacological Reports. 2017.

33. Onoyama K, Kawamata R, Kozai Y, Sakurai T, Kashima I. Comparison of mandibular trabecular structures between normal and diabetic rats: evaluation of spontaneous type 2 diabetes in a rat model. Oral radiology. 2011;27:35.

34. Ay S, Gursoy UK, Erselcan T, Marakoglu I. Assessment of mandibular bone mineral density in patients with type 2 diabetes mellitus. Dentomaxillofac Radiol. 2005;34:32731.

35.Güngör E, Yildirim D, Çevik R. Evaluation of osteoporosis in jaw bones using cone beam CT and dualenergy X-ray absorptiometry. J Oral Sci. 2016;58:185-94.

36. Guerra ENS, Almeida FT, Bezerra FV, Figueiredo PTdS, Silva MAG, Canto GDL, et al. Capability of CBCT to identify patients with low bone mineral density: a systematic review. Dentomaxillofac Radiol. 2017;3:20160475.

37. Hua Y, Nackaerts O, Duyck J, Maes F, Jacobs R. Bone quality assessment based on cone beam computed tomography imaging. Clin Oral Implants Res. 2009;20:767-71.

38. Ibrahim N, Parsa A, Hassan B, van der Stelt P, Aartman IH, Nambiar P. Influence of object location in different FOVs on trabecular bone microstructure measurements of human mandible: a cone beam CT study. Dentomaxillofac Radiol. 2014;43:20130329. 